Philosophie ANTIQUE
Philosophie antique

Problèmes, Renaissances, Usages

13 | 2013

Analogie et connaissance

\title{
Exemple, analogie et paradigme
}

Le paradigmatisme dialectique de Platon

\section{Sylvain Delcomminette}

\section{OpenEdition}

Journals

Édition électronique

URL : https://journals.openedition.org/philosant/904

DOI : $10.4000 /$ philosant.904

ISSN : 2648-2789

Éditeur

Éditions Vrin

\section{Édition imprimée}

Date de publication : 1 novembre 2013

Pagination : 147-169

ISBN : 978-2-7574-0591-8

ISSN : $1634-4561$

Référence électronique

Sylvain Delcomminette, «Exemple, analogie et paradigme », Philosophie antique [En ligne], 13 | 2013, mis en ligne le 01 novembre 2018, consulté le 02 décembre 2022. URL : http://

journals.openedition.org/philosant/904 ; DOI : https://doi.org/10.4000/philosant.904

\section{(c) (i) (3)}

Creative Commons - Attribution - Pas d'Utilisation Commerciale - Pas de Modification 4.0 International - CC BY-NC-ND 4.0

https://creativecommons.org/licenses/by-nc-nd/4.0/ 


\section{EXEMPLE, ANALOGIE ET PARADIGME LE PARADIGMATISME DIALECTIQUE DE PLATON*}

Sylvain DELCOMMINETTE

Université libre de Bruxelles

RÉSUMÉ. Cet article se propose d'étudier les procédés platoniciens de l'exemple, de l'analogie et du paradigme, en insistant à la fois sur leur distinction et sur leur articulation mutuelle. En ce qui concerne l'exemple, il convient d'en distinguer un usage dianoétique, que Platon proscrit, d'un usage proprement dialectique, qu'il encourage et qui peut avoir deux fonctions différentes : faire comprendre une question ou une méthode et faire saisir une structure. L'analogie au sens strict développe cette deuxième fonction, en la complexifiant parfois (notamment dans le Gorgias et la République) par une dimension « constructive ». Reste que le but de l'analogie, comme celui de l'exemple, est avant tout de faire saisir des rapports; et dans la mesure où ces rapports sont purement intelligibles, l'analogie peut elle aussi être considérée comme un procédé dialectique. Enfin, le paradigme tel qu'il est utilisé dans le Sophiste et le Politique développe les traits précédents au sein d'un dispositif très élaboré, qui a pour but essentiel de faire saisir une méthode susceptible d'être appliquée à ce qui fait l'objet de l'examen. En ce sens, il s'agit d'un prolongement de l'un des usages dialectiques des exemples que l'on trouve dès les premiers Dialogues. La pensée paradigmatique de Platon manifeste ainsi une grande continuité, qui repose sur une conception fondamentale : la dialectique, en tant que science suprême qui étudie les Idées, ne peut être décrite de l'extérieur, mais seulement se pratiquer. Cette pratique se prépare, non pas en commençant par se tourner vers le sensible, comme on l'écrit parfois, mais en se tournant vers des cas tout aussi intelligibles - en tant que rapports ou ensembles de rapports, c'est-à-dire structures-, quoique «plus faciles », afin de rendre le dialecticien ultimement capable d'examiner n'importe quel sujet.

SUMMARY. This article examines the Platonic devices of example, analogy and paradigm, in insisting both on their distinction and on their mutual articulation. As for example, one should distinguish its dianoetical use, which Plato proscribes, from its

*. Je remercie l'ensemble des participants au colloque pour leurs questions, remarques et suggestions, et tout particulièrement Leopoldo Iribarren pour ses commentaires écrits qui m’ont incité à corriger et à préciser plusieurs points importants.

Philosophie antique, $\mathrm{n}^{\circ} 13$ (2013), 147-169 
dialectical use, which he promotes and which can have two different functions: clarifying a question or a method and manifesting a structure. Analogy stricto sensu develops this second function, though it sometimes (notably in the Gorgias and the Statesman) makes it more complex by adding a "constructive" dimension. Its aim nevertheless remains to manifest relations; and insofar as these relations are purely intelligible, analogy can itself be deemed dialectical. Finally, paradigm as it is used in the Sophist and the Statesman develops the previous characteristics in a very elaborate device, whose main purpose is to manifest a method which might be applied to the object of investigation. It is therefore a development of the dialectical uses of examples which can be found from the earliest Dialogues onwards. Plato's paradigmatic thought thus demonstrates a great continuity, which is grounded on a fundamental idea: dialectic, being the supreme science of Ideas, cannot be described from the outside, but can only be practised. One can prepare for this practice, not by beginning by looking at sensible things, as is sometimes suggested, but by examining easier cases which are nevertheless intelligible - insofar as they consist in relations or sets of relations, i.e. structures -, in order to make the dialectician ultimately able to examine any object whatsoever. 
Platon est parfois considéré comme le premier théoricien du raisonnement analogique dans la pensée grecque. Cette description me paraît problématique pour deux raisons au moins.

Tout d'abord, parce que je ne crois pas que l'on puisse trouver chez Platon de «théorisation » au sens strict des procédés méthodologiques utilisés dans les Dialogues. De fait, ces procédés relèvent de la dialectique, et la dialectique est selon Platon la science suprême ; or la dialectique est avant tout une pratique, et cette pratique ne peut être décrite de l'extérieur, sinon de manière imagée. Ce n'est pas un hasard si, dans la République, la dialectique est d'abord introduite par le biais d'images, et si, lorsque Glaucon lui demande dans le livre VII d'en décrire le caractère, les espèces et les démarches (532d-e), Socrate se dérobe en répondant: « Mon ami Glaucon, tu ne seras plus à même de m'y suivre (...) c'est que tu ne verrais plus l'image (zikóva) de ce dont nous parlons, mais le vrai lui-même, en tout cas tel qu'il m'apparaît » (533 a $)^{1}$. Ce n'est pas un hasard non plus si, tant dans le Phèdre que dans le Philèbe, la dialectique est introduite de manière mythique, comme faisant l'objet d'une révélation divine. Si la dialectique est la science suprême, elle ne peut être décrite à partir d'un point de vue extérieur qui pourrait prétendre la dominer comme un objet d'examen particulier ${ }^{2}$. Cela ne veut pas dire qu'il est impossible d'en dégager les règles et les principes, mais ceux-ci ne peuvent être mis au jour que de l'intérieur de sa pratique, comme cela se passe, par exemple, dans le Sophiste et le Politique. Je reviendrai sur ce point, qui est capital pour mon propos et constitue le cour même de la pensée paradigmatique de Platon.

L'autre raison pour laquelle cette description me paraît problématique est que l'expression « raisonnement analogique » semble souvent recouvrir des procédés aussi divers que l'analogie stricto sensu (identité de rapports),

1. Les traductions de ce dialogue sont empruntées à Pachet 1993.

2. Sur cette question, voir en dernier lieu Teisserenc 2010, p. 202-216. Je me permets également de renvoyer à Delcomminette 2006, p. 91-96. 
l'exemple, le paradigme, la métaphore et le mythe ${ }^{3}$. Or chez Platon, ces procédés ont des fonctions très différentes les unes des autres, quoique souvent complémentaires. Ce sont ces différences que je voudrais tâcher d'éclairer dans les pages qui suivent. Je laisserai toutefois ici de côté la métaphore et le mythe ${ }^{4}$ pour me concentrer sur l'exemple, l'analogie et le paradigme, en essayant de déterminer ce qui les distingue, mais aussi comment ils s'articulent. Je précise immédiatement que ces distinctions ne recouvrent pas nécessairement des distinctions terminologiques en grec ancien, langue dans laquelle «exemple » et « paradigme » se disent tous deux $\pi \alpha \rho \dot{\alpha}-$ $\delta \varepsilon i \gamma \mu \alpha$. Cela n'empêche pas de repérer des distinctions importantes dans l'usage de ces procédés, à condition précisément de diriger l'examen prioritairement sur celui-ci. Telle est la démarche que je suivrai ici, en essayant de dégager la structure des procédés en question de la manière dont ils sont appliqués dans les Dialogues.

\section{L'exemple}

Dans les Dialogues, nous trouvons tout d'abord une série de passages où Socrate critique l'invocation d'exemples par ses interlocuteurs comme réponse à une question portant sur l'essence ${ }^{5}$. Ainsi, à Lachès qui croit définir le courage par la formule : « Si un homme est prêt à repousser les ennemis tout en gardant son rang, et sans prendre la fuite, sois assuré que cet homme est courageux » (Lachès, 190e $)^{6}$, Socrate objecte qu'il n'a pas répondu à la question qui lui était posée (« Qu'est-ce que le courage ? »), ce qu’il montre au moyen de contre-exemples. De même, à Euthyphron qui déclare que le pieux, c'est ce qu'il est en train de faire à présent, à savoir poursuivre l'auteur d'un crime quel qu'il soit (Euthyphron, 5d-e), Socrate réplique que sa

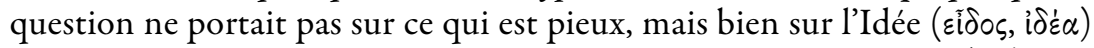
même en vertu de laquelle toutes les choses pieuses sont pieuses (6d). Ou encore, à Ménon qui lui décrit la vertu de l'homme, de la femme, de l'enfant, de l'esclave, etc., Socrate rétorque que sa question ne visait pas un

3. Voir par exemple l'usage très large du terme « analogie » dans Lloyd 1966, qui écrit à la p. 175 : « Here I shall take 'analogy' in its broadest sense, to refer not merely to proportional analogy (a:b::c:d) but to any mode of reasoning in which one object or complex of objects is likened or assimilated to another (of the two particular instances between which a resemblance is apprehended or suggested, one is generally unknown or incompletely known, while the other is, or is assumed to be, better known). 》

4. Sur la métaphore, voir le beau livre de Pender 2000. Sur le mythe, les travaux sont nombreux, mais il manque encore une étude d'ensemble sur la fonction du mythe en général et des mythes particuliers dans les Dialogues de Platon qui tienne compte des dernières avancées dans l'analyse de ce type de discours. Voir cependant Mattéi 1996.

5. Sur ce point, voir en particulier Burnyeat 1977, et surtout Rabbås 2004.

6. Les traductions de ce dialogue sont empruntées à Dorion 1997. 
tel « essaim de vertus », mais bien cette unique vertu, « seule forme caractéristique identique chez toutes sans exception, qui fait d'elles des vertus »

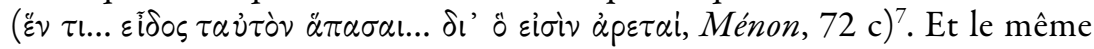
processus se reproduit au début du Théétète à propos de la science (146c-e).

Comme l'a bien montré $\varnothing$. Rabbås ${ }^{8}$, ces «définitions par l'exemple » (ou «définitions par paradigme », comme il les appelle, désignation que je préfère éviter afin de ne pas entraîner de confusion avec le procédé que j'étudierai dans la troisième section ci-dessous) jouaient un rôle essentiel dans la culture grecque du V $V^{e}$ siècle. En effet, celle-ci, parce que l'oralité et la rhétorique y étaient prépondérantes, a développé une éthique fondée beaucoup moins sur des règles et des principes que sur des idéaux et des exemples de manifestations particulièrement éclatantes de la vertu. C'est ce qui explique que de telles définitions soient constamment avancées par les interlocuteurs de Socrate et qu'il soit souvent difficile de faire changer de perspective à ceux-ci. Quel est exactement leur problème aux yeux de Platon ? Pourquoi ne les considère-t-il pas comme le point de départ d'une induction possible, surtout lorsque les exemples sont multiples, comme dans le Ménon et le Théétète ? Ne pourrions-nous pas remonter à l'Idée ellemême en prenant appui sur l'un ou plusieurs de ses participants? Autrement dit, ne pouvons-nous pas viser une Idée à partir d'une de ses images? Comme on le sait, une telle démarche est selon Platon celle des géomètres, qui commencent par tracer la figure qui fait l'objet de leur examen avant de procéder à leurs démonstrations, celles-ci visant non pas la figure en question, mais l'Idée même que cette dernière représente - par exemple le triangle en général, et non pas ce triangle (cf. République, VI, 510b-511b). Pourquoi ne serait-elle pas transposable au type d'interrogation que poursuit Socrate ? Parce que précisément, la question que ne posent pas les géomètres, et à laquelle la géométrie en tant que telle ne peut répondre, est la question «qu'est-ce que ? ». Ils ne s'interrogent jamais sur l'essence même de ce qu'ils examinent, mais se contentent de le poser pour en examiner les propriétés ou les conséquences, en laissant leur point de départ dans l'ombre, comme une simple « hypothèse » (cf. VII, 533b-c). C'est pour cette raison que, s'ils visent bel et bien une Idée, ils n'en atteignent que l'ombre ou le reflet, parce qu'ils ne l'examinent pas en elle-même, dans son essence. Seule la dialectique accomplit cette démarche, mais pour ce faire, elle commence précisément par refuser de prendre appui sur des images afin de viser d'emblée le cœur même de l'objet de son examen par le biais de cette fameuse question $\tau i \dot{\varepsilon} \sigma \tau \imath$;

7. Traduction empruntée à Canto-Sperber 1993a.

8. Rabbås 2004, p. 148-156. 
On peut donc dire que ce que Platon refuse, c'est l'usage que j'appellerais «dianoétique » des exemples, en référence au terme $\delta ı \dot{\alpha} v ı \iota \alpha$ par lequel Socrate désigne la démarche à l'œuvre dans la géométrie. Cet usage résulte en effet d'une confusion, qui consiste à croire que l'image permet d'accéder à l'essence, alors qu'elle ne fait que l'indiquer tout en la recouvrant ou en la maintenant dans l'ombre. En ce sens, je ne peux être d'accord avec la thèse liminaire de V. Goldschmidt dans son ouvrage classique Le Paradigme dans la dialectique platonicienne, selon laquelle «certains passages [des Dialogues] qualifient les sensibles de paradigmes par rapport aux Formes $\gg{ }^{9}$. Ce $\ll$ curieux renversement terminologique $\gg{ }^{10}$ par rapport au paradigmatisme des Idées à l'égard du sensible ne s'observe nulle part dans les Dialogues, et les passages invoqués à l'appui de cette thèse n'impliquent rien de tel - j’y reviendrai. Jamais le sensible ne fournit un « paradigme » à l'intelligible chez Platon, ce qui est bien normal, puisque les Idées ne peuvent être saisies que par la dialectique, qui se distingue de la démarche dianoétique par le fait qu'elle n'utilise pas, quant à elle, d'images sensibles (cf. République, VI, 510b, 511b-c).

Pourtant, cela ne signifie pas qu'il n'existe pas un autre usage possible des exemples qui serait quant à lui pertinent pour la recherche de définitions - bref, un usage proprement dialectique des exemples. On peut même repérer au moins deux usages de ce type : l'un qui vise à faire comprendre une question ou une méthode, l'autre à faire saisir une structure.

Commençons par le premier. Si nous reprenons le Lachès là où nous l'avons laissé, nous voyons que Socrate, afin de mieux faire saisir par Lachès le sens de sa question à propos du courage et le type de réponse qu'il attend, lui explique comment lui-même répondrait à une question du même type portant sur la vitesse (192a-b). Il en va de même dans le Ménon où, après plusieurs essais supplémentaires également infructueux de Ménon en vue de définir la vertu, qui aboutissent une nouvelle fois à fragmenter celle-ci en une multiplicité, Socrate tente de lui expliquer comment procéder en lui proposant des définitions de la figure et de la couleur (74b-76e) à titre d'exemples ( $\pi \alpha p \alpha \delta \varepsilon i \gamma \mu \alpha \tau \alpha, 77 \mathrm{a} 9-\mathrm{b} 1)$. Dans le Théétète, c'est Théétète qui donne un exemple de définition telle que selon lui Socrate la réclame à

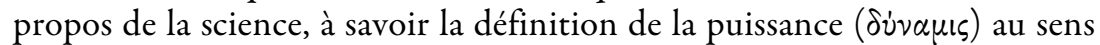
mathématique du terme (147d-148b). Dans tous ces cas, il s'agit d'exemples d'applications d'une méthode de recherche de définition à des objets moins difficiles d'accès et qui dès lors aboutissent à un résultat. De tels exemples ont une double fonction: tout d'abord, certes, une fonction d'exercice (cf. $\mu \varepsilon \lambda \dot{\varepsilon} \tau \eta$, Ménon, 75a9), sur laquelle insiste avec raison 
V. Goldschmidt ${ }^{11}$; mais ensuite, et plus fondamentalement encore, ils visent à faire saisir le sens d'un questionnement et de la méthode à appliquer elle-même. En effet, comme nous l'avons vu, la dialectique ne peut être décrite de l'extérieur, mais seulement de l'intérieur de sa pratique, et tel est le rôle de ces exemples d'application, qui préfigurent sur ce point les grands paradigmes du Sophiste et du Politique, sur lesquels je reviendrai. Ce point est capital : si une Idée ou une essence ne peut être saisie à partir d'un ou de plusieurs exemples de choses qui en participent, la méthode qui permet de l'atteindre, quant à elle, non seulement peut, mais doit être saisie à partir d'exemples de son application.

Tâchons à présent de dégager le deuxième usage dialectique des exemples. Si nous revenons en arrière dans le Lachès, nous voyons Socrate commencer par s'appuyer sur les exemples de l'examen du remède pour les yeux et du mors pour le cheval afin de faire saisir à ses interlocuteurs que « lorsqu'on examine une chose en vue d'une autre chose, la délibération porte sur ce en vue de quoi on a entrepris l'examen, et non sur ce qu'on cherchait en vue d'une autre chose » (185d). Dans un deuxième temps, il transpose cette règle générale à l'examen actuel, qui porte sur l'éducation à inculquer en vue de l'âme. Nous avons ici un cas très clair d'induction: partant de deux exemples, on remonte à la règle générale, puis on subsume un cas particulier différent des deux exemples précédents sous cette règle générale. On retrouve cette structure de raisonnement dans de nombreux autres passages des Dialogues. Lisons par exemple cette page du Gorgias:

Socrate: Quand on a bien appris l'art du maçon, on devient maçon, n'estce pas?

Gorgias : Oui.

Socrate : Et quand on a appris la musique, on devient musicien ?

Gorgias : Oui.

Socrate : Et médecin, si on a appris la médecine; d'ailleurs, on peut faire la même remarque pour les autres formations: quand on a suivi un apprentissage, le savoir qu'on a appris, n'a-t-il pas déjà, sur soi, comme sur son propre ouvrage, imprimé sa marque ?

Gorgias : Oui, parfaitement.

Socrate : Donc, le même argument s'applique aussi à la justice : quand on a bien appris la justice, on est devenu juste.

Gorgias : Il n'y a aucun doute là-dessus. (Gorgias, $460 b^{12}$.)

Ce raisonnement a exactement la même structure que le précédent, sinon qu'il s'appuie sur trois exemples plutôt que deux. Le nombre d'exemples d'où l'on part n'est d'ailleurs pas déterminant, et dans certains cas,

11. Ibid., p. 16

12. Les traductions de ce dialogue sont empruntées à Canto-Sperber 1993b. 
Socrate demande s'il peut faire l'économie d'une énumération plus longue et passer directement à la règle générale (voir notamment Gorgias, 496d). Dans d'autres textes, Socrate commence par énoncer la règle générale avant de l'illustrer au moyen d'exemples (voir notamment Gorgias, 476b-d).

Dans chacun de ces passages, Socrate s'éloigne de l'objet immédiat de la recherche pour examiner des cas apparemment triviaux et anodins, d'où il tire une loi qu'il applique au premier objet. C'est ce type de procédé qui suscite l'exaspération d'un Calliclès: « Ah oui, vraiment, par les dieux, tu ne parles jamais que de cordonniers, de cardeurs, de bouchers, et de médecins, tu ne pourras donc jamais t'en empêcher! Comme si c'était de ces gens-là que nous discutions! » (Gorgias, 491a.) Dans le Banquet, Alcibiade semble répondre à Calliclès lorsqu'il prononce l'éloge de Socrate dans les termes suivants :

Qu'on veuille bien, en effet, écouter les discours de Socrate : à la première impression, on ne manquera pas sans doute de les trouver absolument ridicules. Tels sont les mots, les phrases qui en sont l'enveloppe extérieure, qu'en vérité on dirait la peau d'un insolent satyre! Car il vous y parle d'ânes bâtés, de forgerons, de cordonniers, de corroyeurs; il a toujours l'air de se répéter, dans ses expressions comme dans ses pensées; si bien qu'il n'y a pas au monde d'ignorant ou d'imbécile qui ne fasse de ses discours un objet de dérision. Mais arrive-t-il qu'on les voie s'entrouvrir et qu'on en arrive à l'intérieur, alors on commencera de les trouver, dans le fond, pleins d'intelligence (voũv घ̈ $\chi 0 v \tau \alpha \varsigma$ ), et les seuls qui soient tels. (Banquet, 221e-222 $\mathrm{a}^{13}$.)

Mais en quoi cet usage des exemples par Socrate differe-t-il de l'usage qu'il proscrit dans la recherche de définitions ? En ce qu'il ne consiste pas à partir d'un cas particulier pour viser à travers lui l'essence dont il participe, mais bien plutôt à partir d'un (ou plusieurs) rapport(s) entre au moins deux termes pour le $(\mathrm{s})$ transposer à d'autres termes par l'intermédiaire de la loi générale qui les subsume comme cas particuliers. Le nœud de la différence réside en ce que le point de départ n'est pas ici un terme unique, mais un rapport entre deux termes (ou plus) : par exemple, l'examen d'une chose en vue d'une autre ou le rapport entre un art et le sujet qui l'a appris. Or ce rapport n'est pas, quant à lui, sensible, en aucun sens possible du terme : il ne peut être saisi que par l'intelligence (voũs). Dans les cas de ce genre, il ne s'agit donc pas de passer d'une image (sensible) à l'Idée correspondante, mais d'un rapport intelligible à un autre rapport tout aussi intelligible. L'affirmation d'Alcibiade selon laquelle les discours de Socrate sont «pleins d'intelligence » pour qui sait les pénétrer en profondeur doit être comprise 
littéralement : avec cet usage des exemples, nous nous situons au cœur de l'activité dialectique.

Notons que cela ne place pas pour autant ce type de raisonnement audelà de tout soupçon, et qu'il demeure possible de le contester. C'est ce qui arrive dans le Charmide où, à Socrate qui rapproche la sagesse ( $\sigma \omega \phi p o \sigma i v \eta)$ de la médecine, de l'architecture et des autres arts $(\tau \dot{\varepsilon} \chi \nu \alpha \iota)$ et demande ce que la première produit, Critias répond :

Mais tu ne cherches pas comme il faut, Socrate (...). En effet, la sagesse est

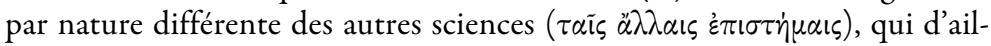
leurs ne se ressemblent pas non plus entre elles. Or toi tu conduis ton enquête comme si elles étaient semblables ! Eh bien, dis-moi, demanda-t-il, ce que le calcul ou la géométrie produit de comparable à la maison, qui est l'œuvre de l'architecture, ou au manteau, qui est l'œuvre du tissage, ou aux autres œuvres de ce genre que l'on pourrait citer en grand nombre et qui sont produites par les nombreuses techniques. Peux-tu donc me montrer quelle œuvre de ce genre produisent ces deux sciences ? Tu en seras bien incapable. » $\left(166 \mathrm{a}-\mathrm{b}^{14}\right.$.)

Ce que Socrate approuve : si l'on comprend les arts et les sciences comme étant identiques, comme le fait manifestement Critias ici, alors effectivement, rien n'autorise à appliquer le rapport entre un art et son produit à tous les arts, mais seulement à ceux d'entre eux qui relèvent de la production (quant à savoir si Critias a raison de penser que la sagesse ne relève pas de cette dernière, c'est une autre question). Ici, l'usage d'exemples, plutôt que de permettre la saisie d'une ressemblance entre différents rapports qu'il serait dès lors possible de rassembler au sein d'un même genre, a pour effet de mettre en évidence une différence qui implique une division du genre en question.

Le même processus s'opère au début du Gorgias, lorsque Socrate tâche de faire préciser par Gorgias ce qui distingue la rhétorique des autres arts, dont il établit par la voie d'exemples (la médecine, la gymnastique, etc.) qu'ils peuvent eux aussi être considérés comme portant sur les discours, du moins les discours relatifs à l'objet dont ils s'occupent. Gorgias répond « qu'en tous ces autres arts, toute la connaissance, on peut le dire, se rapporte à une action manuelle ou une activité de même genre. Mais la rhétorique, elle, ne consiste aucunement en un travail manuel. Au contraire, dans toute son action, dans l'exécution de sa tâche, le discours est seul instrument $\gg(450 \mathrm{~b}-\mathrm{c})$. Or dans la suite, Socrate va reprendre cette distinction en montrant qu'elle est peut-être pertinente, mais pas suffisante, car d'autres arts, par exemple l'arithmétique, le calcul ou la géométrie, voire les 
jeux de pions, sont dans le même cas. Ici encore, l'usage d'exemples sert à mettre en évidence une différence qui entraîne la division du genre considéré - peu importe que tel soit le but initial de Socrate ou non.

Un tel usage des exemples n'est pas moins dialectique que le premier; au contraire, il permet de rendre manifeste que les exemples sont pertinents pour les deux grands mouvements de la dialectique : perception des ressemblances et mise au jour des différences ; rassemblement au sein d'un genre et division de ce genre en espèces. La méthode de rassemblement et de division, thématisée et pratiquée dans les Dialogues plus tardifs à partir du Phèdre, n'est que la systématisation de ce double mouvement présent dès les premiers Dialogues, et il n'est pas étonnant qu'elle s'appuie elle aussi constamment sur l'invocation d'exemples, sans que cela implique le moindre « basculement dans l'empirique », comme on l'a parfois écrit à tort ${ }^{15}$.

Nous avons donc repéré deux usages légitimes des exemples dans le cadre de la démarche socratique de recherche de définitions. Selon le premier, les exemples servent à faire saisir une méthode ; selon le second, ils visent à faire saisir un rapport ou un ensemble de rapports - bref, une structure -, soit pour l'appliquer, soit pour le distinguer de l'objet de la recherche. C'est cette deuxième fonction que nous retrouverons dans le cas de l'analogie, sur laquelle nous allons nous pencher à présent.

\section{L'analogie}

Comme le rappelle R. Robinson, l'analogie peut être définie comme une induction qui passe d'un cas particulier à un autre sans mentionner l'universe ${ }^{16}$. Il faut toutefois préciser que, de même que l'induction n'est admise par Platon qu'en tant qu'elle permet de passer d'un (ou de plus d'un) rapport particulier entre deux termes au moins à la loi générale sous laquelle ce rapport est subsumé, l'analogie passe directement d'un cas particulier de rapport à un autre. D'où la conception classique de l'analogie comme identité de rapports, qui correspond au sens mathématique du terme à $\nu \alpha \lambda o \gamma i \alpha$ tel qu'il est défini en Timée, 31c-32a. Bien que Platon utilise très peu les termes de cette famille en dehors de leur contexte strictement mathématique (à l'exception de deux passages célèbres de la République: VI, 508b13 et VII, 534a6), l'usage de l'analogie comme identité de rapports est quant à lui constant dans les Dialogues. Il se fonde sur les mêmes raisons que le deuxième usage dialectique des exemples que nous venons de relever : simplement, l'identité de deux (ou de plus de deux) rapports est saisie immédiatement, sans passer par la formulation de la loi gé-

15. Sur l'usage des exemples dans la méthode de rassemblement et de division, je me permets de renvoyer à Delcomminette 2000, p. 95-109.

16. Robinson 1953, p. 207. Cf. Aristote, Premiers Analytiques, II, 24, 69a13-19. 
nérale. Par exemple, dès le début du Gorgias, Chéréphon interroge Polos de manière analogique :

Chéréphon : Bon, voici ma question. Si Gorgias connaissait le même art que son frère, Hérodicos, comment serait-il correct de l'appeler ? comme son frère, n'est-ce pas?

Polos: Oui, absolument.

Chéréphon : On aurait donc raison de dire qu'il est médecin.

Polos : Oui.

Chéréphon: Mais s'il connaissait le même art qu'Aristophon, fils d'Aglaophon, ou que, d'ailleurs, le frère d'Aristophon, comment serait-il juste de l'appeler ?

Polos : Peintre, c'est évident.

Chéréphon: Mais alors quel est l'art que Gorgias connaît ? de quel juste nom faut-il l'appeler ? (Gorgias, 448b-c.)

Chéréphon se montre ici bon disciple de Socrate: le procédé qu'il utilise est exactement le même que ceux que nous avons repérés plus haut, sinon qu'il ne prend pas la peine d'énoncer la règle générale (à savoir que le détenteur d'un art est nommé d'après le nom de l'art dont il est détenteur) avant de passer au cas particulier qui l'intéresse. Socrate fait exactement la même chose un peu plus loin, lorsqu'il interroge Gorgias sur l'objet de la rhétorique :

Socrate : Mais la rhétorique ? Sur quoi porte-t-elle ? Quel est son objet ? Par exemple, le tissage, lui, est en rapport avec la confection des vêtements, n'est-ce pas?

Gorgias : Oui.

Socrate: Et la musique, elle, se rapporte-t-elle bien à la composition des chants?

Gorgias : Oui.

Socrate: (...) Eh bien, allons, réponds-moi aussi bien, au sujet de la rhétorique, cette fois. Quel est l'objet que la rhétorique fait connaître ?

Gorgias : Les discours. (Gorgias, 449 d-e.)

Ici encore, la loi générale, selon laquelle tout art a un objet, n'est pas mentionnée.

On pourrait multiplier les exemples. Mais quelle est dans ces conditions la particularité des grandes analogies que l'on trouve dans le Gorgias et la République, à savoir respectivement celle qui vise à mettre au jour la nature de la rhétorique et celle qui est établie entre la cité et l'âme humaine ?

Commençons par l'analogie du Gorgias. Ce qui frappe d'emblée dans le long passage qui vise à faire de la rhétorique l'analogue pour l'âme de la cuisine pour le corps et la contrefaçon de la justice (463a-465d), c'est sa complexité. Il ne s'agit plus simplement, comme dans les passages précédemment mentionnés, de dégager un rapport d'un cas particulier où il 
apparaît avec plus d'évidence pour le transposer à un autre cas où il est moins manifeste, mais de construire un ensemble de rapports dans un certain contexte pour organiser sur son modèle un autre contexte afin d'éclairer la situation qu'y occupe l'objet de la recherche. En l'occurrence, c'est la double division de la $\tau \dot{\varepsilon} \chi \chi \nu \eta$ - selon qu'elle s'adresse au corps ou à l'âme, d'une part, et selon qu'elle entretient et stabilise un état sain (gymnastique pour le corps, législation pour l'âme) ou qu'elle corrige un état corrompu (médecine pour le corps, justice pour l'âme), d'autre part -, qui fournit le modèle sur lequel peut également être divisé ce savoir-faire

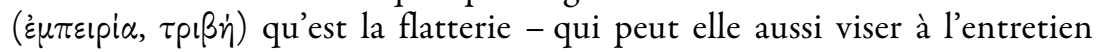
(apparent) du corps (cosmétique) ou de l'âme (sophistique), ou avoir des visées correctrices (également apparentes) en ce qui concerne le corps (cuisine) ou l'âme (rhétorique). D'où les analogies suivantes : la rhétorique est à la justice (dans le domaine de la correction) ce que la sophistique est à la législation (dans le domaine de l'entretien), c'est-à-dire, de manière générale, ce que le savoir-faire est à l'art; ou encore, la rhétorique est à la cuisine ce que l'âme est au corps. S'il y a bien, dans ces deux équivalences, un rapport qui est plus général que les autres (le rapport du savoir-faire à l'art, ou celui de l'âme au corps), celui-ci n'est plus traité comme la loi générale qui fonderait l'équivalence entre les rapports particuliers, mais bien plutôt comme l'un des termes de l'équivalence, qui donne son sens à celleci, précisément par sa généralité. Enfin, il faut noter que l'analogie n'est pas en tant que telle un procédé démonstratif et ne fonctionne pas seule : si elle permet de définir la rhétorique (comme flatterie de l'ordre du savoir-faire qui s'adresse à l'âme et a des prétentions correctrices), c'est seulement en ce qu'elle oriente la division, qui est le véritable procédé définitionnel. Quant à la vérité de cette définition (différente de sa validité formelle), elle n'est pas encore établie par là : tout ce passage vise seulement à manifester la manière

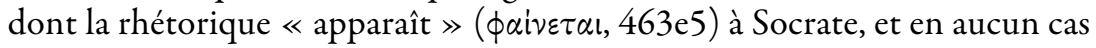
une définition qui soit au-delà de toute contestation possible. La structure mise au jour par l'analogie a certainement une validité en tant que telle, mais cela ne suffit pas à garantir la vérité de son application à l'objet étudié.

Ces caractéristiques se rencontrent également dans l'analogie entre la cité et l'âme humaine que l'on trouve dans la République ${ }^{17}$. Là aussi, le modèle grâce auquel la structure de l'âme humaine va être mise au jour est construit, puisqu'il s'agit d'une cité «idéale », qui n'existe nulle part ailleurs que « dans les logoi », et dont l'élaboration s'étend du livre II, 368c, au livre IV, 434d. Là non plus, l'égalité de rapports entre la structure de la cité et celle de l'âme humaine n'est pas fondée dans une loi générale à

17. Ce thème a fait l'objet de nombreuses études qui dépassent largement mon propos actuel. Pour une présentation stimulante des problèmes en présence, voir Ferrari 2003. 
laquelle toutes deux seraient subordonnées : «l'élément commun » qui permet leur rapprochement est bien plutôt la justice, c'est-à-dire précisément une certaine structure (un certain rapport harmonieux des parties) qui est susceptible d'être trouvée en chacune des deux et qui est explici-

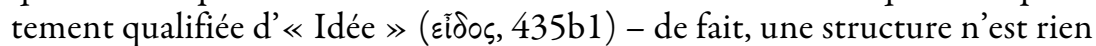
de sensible, et la justice comme vertu consistant à accomplir sa fonction propre ne peut être qu'intelligible. Enfin, là non plus l'analogie n'est pas démonstrative par elle-même, mais fournit seulement un guide heuristique dont les résultats doivent être vérifiés indépendamment. C'est ce que reconnaît explicitement Socrate :

Mais pour l'instant nous allons mener à son terme le type d'examen qui nous a fait espérer que si nous commencions par essayer de considérer la justice en quelque réalité plus grande qui la possède, il serait ensuite plus facile de distinguer ce qu'elle est dans un individu humain. Et il nous a alors semblé que cette réalité, c'était une cité, et ainsi nous avons fondé la meilleure cité que nous pouvions, sachant bien que c'est dans la bonne cité que la justice pourrait se trouver. Dès lors, ce que la justice nous est apparue être dans la cité, transférons-le (่̇ $\pi \alpha \nu \alpha \phi \dot{\varepsilon} p \omega \mu \varepsilon \nu)$ à l'individu; et si cela est reconnu être la justice, tout ira bien. Si en revanche elle apparaît être quelque chose d'autre dans l'individu, revenant à nouveau à la cité, nous la mettrons à l'épreuve; et peut-être qu'en confrontant ces réalités et en les frottant l'une contre l'autre, comme on fait avec un briquet, nous arriverons à faire jaillir la lumière de la justice. (République, IV, 434d-435a.)

La fin de ce passage est intéressante, car elle suggère qu'une méthode alternative pourrait être adoptée en cas d'échec de l'analogie. La nature de cette méthode est extrêmement difficile à déterminer, puisque, comme on le sait, l'analogie permettra en l'occurrence bel et bien de dégager la justice dans le cas de l'individu également. Socrate semble suggérer qu'une divergence entre la cité et l'individu pourrait elle aussi nous mettre sur la bonne voie en nous contraignant à les confronter pour « faire jaillir la lumière ». Cette situation pourrait sans doute être rapprochée de celles du Charmide et du Gorgias analysées plus haut, où la confrontation entre des exemples différents suscitait la perception d'une différence plutôt que d'une ressemblance.

Quoi qu'il en soit, ce passage montre clairement que l'analogie n'est pas autosuffisante et doit s'allier à d'autres procédés pour donner lieu à des résultats. Dans le livre IV de la République, il conviendra d'abord de montrer que l'âme humaine, comme la cité qui vient d'être constituée, est composée de trois parties. Il s'agit là d'un présupposé de l'analogie que celleci ne suffit aucunement à établir par elle-même. De fait, la tripartition de l'âme fera l'objet d'une argumentation indépendante reposant sur des principes propres, en particulier une variante du principe de contradiction (cff. 
436b-c) et ce que l'on peut appeler le principe de la détermination réciproque des corrélatifs (438a-b), sur la base desquels sont ensuite analysées diverses situations de conflits moraux ${ }^{18}$. Une fois la tripartition de l'âme établie, les conditions de l'application de l'analogie seront réunies; restera alors à examiner si les définitions des vertus dégagées de l'analyse de la cité demeurent pertinentes dans le cas de l'âme ainsi conçue. C'est seulement au terme de cet examen, dont le résultat s'avérera positif, que la correspondance entre les vertus dans l'âme et dans la cité sera affirmée de manière catégorique $(c f . \grave{\alpha} \nu \alpha \gamma \kappa a i o v, 441 c 8)^{19}$. On le voit, comme dans le Gorgias, l'analogie fournit un cadre général qui oriente la recherche, mais elle ne suffit pas par elle-même : elle doit être complétée par d'autres procédés pour aboutir à un résultat pleinement satisfaisant.

De ce qui précède, nous pouvons conclure que l'analogie a toujours pour but de transférer un certain rapport ou une certaine structure d'un objet à un autre, et que de ce point de vue, elle n'a elle-même affaire qu'à de l'intelligible. Pas plus que dans le cas de l'exemple, nous ne nous trouvons ici face à ce fameux « renversement » du paradigmatisme des Idées cher à Goldschmidt, selon lequel ce serait le sensible qui deviendrait le paradigme de l'intelligible. Le procédé de l'analogie sert à passer de l'intelligible à l'intelligible, et c'est précisément pour cette raison qu'il est un instrument à part entière de la dialectique - un instrument important, certes, mais qui demande toutefois à être complété par d'autres. La différence avec l'exemple, du moins dans le cas des grandes analogies du Gorgias et de la République, est que les modèles d'où sont dégagées les structures qui seront dans un second temps transposées à l'objet de la recherche sont ici construites et non simplement «constatées ». Nous allons voir que cet aspect de la méthode va être encore renforcé dans le procédé dialectique le plus complexe utilisé par Platon, à savoir le paradigme.

\section{Le paradigme}

Par « paradigme », j'entends ici le procédé dialectique utilisé dans le Sophiste et surtout le Politique, où il fait l'objet d'une importante discussion. Dans le Sophiste, on le sait, le paradigme utilisé afin de préparer l'enquête sur le sophiste est celui de la pêche à la ligne; dans le Politique, celui du tissage.

Qu'est-ce qui distingue ce procédé des précédents ? Fondamentalement, on peut dire qu'il ne fait que reprendre chacun des traits mis au jour jusqu'à présent, mais en les développant et en les intégrant dans une méthode unique beaucoup plus sophistiquée. En ce sens, on peut dire qu'il repré-

18. J'ai examiné cette argumentation dans Delcomminette 2008.

19. Je remercie Gábor Betegh d'avoir attiré mon attention sur ce passage. 
sente à la fois la synthèse et le sommet des procédés dialectiques étudiés dans les pages précédentes.

(1) Premièrement, en continuité avec le premier usage dialectique de l'exemple repéré ci-dessus, le paradigme vise avant tout à faire saisir une méthode. Dans le Sophiste, c'est afin de faire saisir la méthode de rassemblement et de division en vue de donner le logos de l'objet d'examen que l'Étranger propose à Théétète de s'exercer sur un sujet « plus petit » :

L'Étranger : (...) Quant à la race qui fait l'objet de notre enquête, ce n'est point la tâche la plus facile de comprendre ce que c'est que le sophiste! Mais, quelque grandes œuvres qu'il faille mener à bonne fin, la règle admise, en ce cas, par tous et de tout temps, c'est qu'il s'y faut d'abord essayer sur des exemples réduits et plus faciles avant que d'aborder en eux-mêmes les

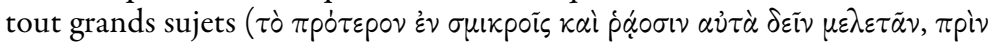

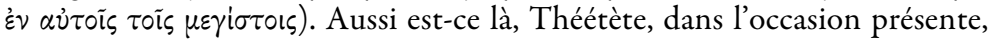
le parti que je conseille pour nous deux : avant cette difficile et pénible chasse qu'exigera, nous le savons, le genre sophistique, faire d'abord, sur quelque sujet plus facile, l'essai de la méthode applicable à cette recherche

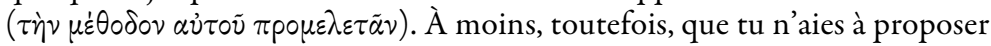
quelque voie plus aisée.

Théétète : C'est que je n'en ai point.

L'Étranger: Veux-tu donc que nous fassions l'investigation de quelque sujet simple en essayant d'y trouver un paradigme $(\pi \alpha \rho \dot{\alpha} \delta \varepsilon \varepsilon \gamma \mu \alpha)$ pour notre grand sujet?

Théétète : Oui. (Sophiste, $218 \mathrm{c}-\mathrm{e}^{20}$.)

Dans ce texte, « plus petit » ou « moins grand » sont avant tout à entendre au sens de «plus facile » : c'est parce que l'objet qui sera choisi comme paradigme demande moins d'efforts et de perspicacité pour être mis au jour qu'il permettra à un dialecticien moins expérimenté de se concentrer sur la méthode qui sera appliquée, afin de la transposer dans un deuxième temps sur un objet plus complexe ${ }^{21}$. Par ailleurs, si la dimension d'exercice, bien mise en évidence par Goldschmidt, est ici fortement soulignée, elle n'est qu'un aspect de la question : en effet, la recherche concernant le pêcheur à la ligne qui va suivre est en même temps la première introduction et présentation de cette méthode, du moins pour Théétète. Ceci confirme l'une de mes remarques liminaires: la dialectique, en tant

20. Les traductions de ce dialogue sont empruntée à Diès 1925, parfois légèrement modifiées.

21. Contrairement aux apparences, il en va de même dans l'analogie de la République, où, certes, il s'agit de chercher la justice dans quelque chose de plus grand (la cité) pour s'aider à la retrouver dans quelque chose de plus petit (l'âme humaine), mais précisément parce que cela est plus facile ( $\dot{\rho} \tilde{\alpha}, 0 v, 434 \mathrm{~d} 7$ ) : la grandeur dont il est question alors n'a pas le même sens que dans ce texte du Sophiste, mais le principe sur lequel repose la méthode invoquée est identique. 
que science suprême, ne peut être décrite de l'extérieur, mais seulement de l'intérieur de sa pratique.

Cet aspect est encore exacerbé dans le Politique, où l'Étranger, voulant introduire un paradigme afin de relancer et de compléter la recherche à propos du politique, dit avoir besoin d'un paradigme pour expliquer ce qu'est le paradigme en général $(277 \mathrm{~d})$. C'est là plus qu'un jeu de mots : on peut y voir le refus de toute théorie de la méthode élaborée à partir d'un point de vue extérieur. Le paradigme, en tant que procédé dialectique, ne peut lui-même être saisi qu'à partir de l'une de ses applications. En l'occurrence, c'est l'apprentissage des lettres qui va fournir le «paradigme du paradigme », en ce que celui-ci met en jeu la confrontation de certaines syllabes dans lesquelles apparaît telle lettre sans que l'élève soit capable de l'y repérer avec d'autres syllabes dans lesquelles elle apparait également et où il la repère, de manière que ce rapprochement lui permette de transposer la reconnaissance de ces lettres des secondes aux premières (277e-278c). On le voit, le paradigme du paradigme vise avant tout à nous faire saisir une méthode. Cependant, dans le cas des lettres, cette méthode est appliquée à des choses sensibles (lettres et syllabes); il s'agit donc de la transposer dans le domaine de l'intelligible pour la transformer en véritable méthode dialectique, où elle est confrontée non plus aux lettres et aux syllabes sensibles, mais aux éléments « de toutes choses » et aux complexes qu'ils constituent (278c-d) - bref, aux Idées et à leurs entrelacements. Pour ce faire, nous allons avoir besoin d'un nouveau paradigme, qui ait cette fois pour objectif de nous montrer comment l'application du procédé paradigmatique en général va s'opérer dans le cas particulier qui nous occupe, à savoir la définition du politique. Ce paradigme est celui du tissage, dont l'Étranger va montrer qu'il ne suffit pas de le définir par ses relations avec les arts qui lui sont apparentés (c'est-à-dire qui appartiennent au même genre), mais qu'il faut également déterminer ses rapports avec tous les arts qui interviennent dans ce que l'on peut appeler sa « sphère d'activité », c'est-à-dire tous les arts qui contribuent à des niveaux différents au tissage de la laine sans pour autant s'y identifier - en préparant ses outils ou le matériau qu'il va utiliser, par exemple (279a-283a). Le même art (la même lettre) doit donc être repéré dans deux contextes différents (deux syllabes différentes), et sa reconnaissance dans le deuxième contexte (sa sphère d'activité), problématique à ce stade, doit prendre appui sur sa reconnaissance dans le premier contexte (la définition du tissage), d'ores et déjà atteinte quant à elle par l'application de la méthode de rassemblement et de division. C'est la même démarche qu'il s'agira alors d'appliquer au politique, en prenant appui sur la première définition qui en a été atteinte dans la première partie du dialogue (258b-267c), qui, loin d'être rejetée comme on le dit souvent, 
sert de guide à l'entreprise de distinction du politique de ses rivaux dans la suite du dialogue $\left(287 \mathrm{a}-305 \mathrm{e}^{22}\right)$.

(2) Deuxièmement, de même que les exemples dans leur second usage dialectique, le paradigme vise à faire saisir des rapports. Ici, cette finalité n'est pas indépendante de la première - faire saisir une méthode -, dans la mesure où la méthode en question est précisément une méthode de mise en rapport. La méthode de rassemblement et de division dont l'examen de la pêche à la ligne fournit le paradigme dans le Sophiste est une méthode qui vise à définir un art en le distinguant de tous les autres, rassemblés au sein du genre de départ. Définir, c'est établir des ressemblances et des différences, c'est-à-dire mettre en relation; et ce n'est bien entendu pas un hasard si l'entreprise de définition du sophiste conduira à une mise en évidence du problème de la «communauté des genres» et de ces fameux « genres-voyelles » (l'être, le même et l'autre) qui rendent possible la mise en relation des Idées entre elles. Quant au paradigme du Politique, il vise à faire fond sur cette première mise en relation d'un élément (en l'occurrence, l'art du tissage ou l'art politique) avec les autres éléments appartenant à un même complexe (en l'occurrence, les « arts parents », c'est-àdire ceux qui appartiennent au même genre) pour analyser la relation entre ce même élément et les autres éléments appartenant à un autre complexe (en l'occurrence, la sphère d'activité de l'art en question). Le modèle est donc, à proprement parler, non pas l'art lui-même, mais bien plutôt la structure dégagée de son analyse, à savoir la manière dont la définition d'un art considéré en lui-même permet également de le distinguer de tous les autres arts avec lesquels il est amené à interagir. Si une telle structure peut être transposée à tous les arts, ou du moins à tous les arts d'un certain type, c'est précisément parce qu'elle est une pure articulation de rapports. En ce sens, il est une nouvelle fois erroné de dire que dans les cas de ce genre, ce serait le sensible qui deviendrait paradigme de l'intelligible : non seulement parce que l'art de la pêche à la ligne ou du tissage n'est ni plus ni moins sensible que la sophistique ou l'art politique, mais plus fondamentalement encore parce que ce n'est pas l'art lui-même, mais la méthode dont il fait l'objet et la structure que son application permet de mettre au jour qui servent à proprement parler de modèles, et que celles-ci n'ont bien entendu rien de sensible.

(3) Troisièmement, comme les grandes analogies du Gorgias et de la République, les paradigmes du Sophiste et du Politique sont construits. Cette caractéristique est une nouvelle fois intrinsèquement liée à la première, à savoir qu'il s'agit avant tout de faire saisir une méthode par la pratique de celle-ci : le paradigme choisi doit donc, certes, être plus facile, mais néan-

22. Je résume ici les analyses que j’ai développées dans Delcomminette 2000, p. 225-258 et 273-304. 
moins réclamer une application un tant soit peu élaborée de la méthode en question. Celle-ci est particulièrement développée dans le Politique, à tel point que l'Étranger en vient à son terme à s'interroger sur la pertinence de ce développement et à se demander s'il n'était pas trop long. On connaît sa réponse : la vraie norme dans un cas de ce genre est le gain dialectique, car toute l'enquête a pour principal objectif de nous rendre «plus dialecticiens $\gg(\delta เ \alpha \lambda \varepsilon \kappa \tau \imath \kappa \omega \tau \dot{\varepsilon} p o l, 285 \mathrm{~d} 7,287 \mathrm{a} 3)$, ce qui veut dire également

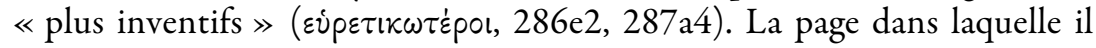
fait cette déclaration mérite d'être citée en entier, car elle constitue certainement le fondement et la justification les plus clairs de la pensée paradigmatique de Platon :

L'Étranger : Suppose qu'on nous fasse cette question : dans les classes où l'on apprend à lire, quand on demande à quelqu'un de quelles lettres est fait tel ou tel mot, ne le fait-on ainsi chercher que pour l'amener à résoudre ce problème particulier, ou bien pour le rendre plus apte à résoudre tous les problèmes possibles de grammaire?

Socrate le Jeune : Tous les problèmes possibles, évidemment.

L'Étranger : Que dirons-nous alors de notre enquête au sujet du politique ? Est-ce directement par intérêt pour lui que nous nous la sommes imposée, ou bien est-ce pour devenir meilleurs dialecticiens sur tous les sujets possibles?

Socrate le Jeune : Ici encore, évidemment, pour la formation générale.

L'Étranger : D'ailleurs, on peut bien le dire, se mettre en quête d'une définition du tissage par amour pour le tissage lui-même, aucun homme de bon sens n'y consentirait. Mais je crois qu'il échappe à la plupart des gens

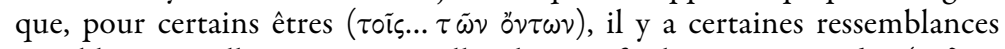
sensibles naturelles grâce auxquelles ils sont faciles à comprendre ( $\dot{p} \alpha \delta i \omega s$

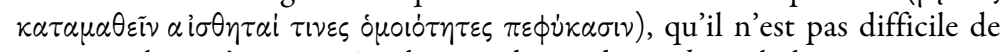
montrer, lorsqu'on veut, à celui qui demande un logos, le lui exposer sans compliquer les choses et indépendamment d'un logos; mais que pour les

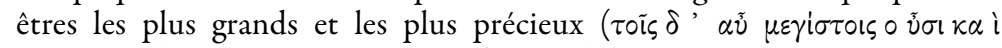

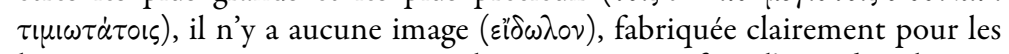
hommes, que pourrait montrer celui qui veut satisfaire l'âme de celui qui interroge et qui, adaptée à l'un des sens, la satisferait suffisamment. C'est pourquoi il faut s'exercer à être capable de donner et de recevoir le logos de chaque chose : en effet, les incorporels, qui sont les plus beaux et les plus grands, peuvent seulement être montrés clairement par le logos, et par rien d'autre, et ce sont ceux-ci que vise tout ce que nous disons maintenant. Mais pour tout sujet, l'exercice est plus facile s'il porte sur de petits <exem- 
ples> plutôt que sur des grands ( $\dot{\rho} \alpha \dot{\alpha} \omega \nu \delta$ ' $\dot{\varepsilon} \nu \tau 0 \tilde{i} \varsigma \dot{\varepsilon} \lambda \dot{\alpha} \tau \tau 0 \sigma \nu \nu \dot{\eta} \mu \varepsilon \lambda \dot{\varepsilon} \tau \eta \pi \alpha \nu \tau \dot{c} \varsigma$

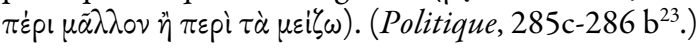

Les dernières lignes de ce texte (285d10-286b1) ont fait couler beaucoup d'encre, notamment suite à un article de G. E. L. Owen consacré à l'« irreprésentable ${ }^{24}$. » La plupart des interprétations en présence me paraissent toutefois souffrir d'un double défaut : d'une part, elles ne tiennent pas suffisamment compte du contexte immédiat du passage, notamment des quelques lignes qui précèdent à propos de l'apprentissage des lettres, et d'autre part, elles identifient indûment les « petits exemples » de la dernière ligne avec les êtres qui ont des ressemblances sensibles naturelles. Une fois ces deux écueils évités, le sens de ce texte me semble assez clair :

- Certains êtres présentent des ressemblances sensibles naturelles qu'il suffit de montrer pour en rendre compte de manière aisée. Ces êtres ne peuvent être que les choses sensibles, car en aucun cas, la monstration d'une image sensible ne peut prétendre rendre compte d'une Idée quelle qu'elle soit : au contraire, nous avons vu que cet usage des images correspondait à l'usage des exemples proscrit par Socrate dès les premiers Dialogues. Même si certaines Idées peuvent apparaître plus manifestement dans le sensible (le beau, par exemple : voir Phèdre, 250b-d), il ne suffit pas de présenter leurs images pour satisfaire l'âme qui s'interroge à leur sujet : les remontrances de Socrate dans l'Hippias majeur le prouvent à suffisance. Mais à quoi l'Étranger fait-il référence? C'est ici qu'il convient de garder à l'esprit le rappel du paradigme des lettres qui figure juste avant ces fameuses lignes : là, nous avons un exemple de «monstration » qui vise à enseigner quelque chose à quelqu'un en lui faisant voir les « ressemblances sensibles naturelles, faciles à comprendre ». D'après la description précédente, en effet, c'est exactement de cette manière que procèdent les maîtres d'école : afin d'enseigner les lettres (sensibles : écrites ou prononcées), on rapproche des syllabes (également sensibles) dans lesquelles elles apparaissent pour mettre en évidence les ressemblances entre certains de leurs éléments. Cette monstration suffit, parce que ce que l'on cherche à enseigner est d'ordre sensible (les lettres dites ou écrites).

23. La traduction des lignes $285 \mathrm{~d} 10-286 \mathrm{~b} 1$ se veut la plus littérale possible ; elle s'inspire en particulier des traductions de M. Dixsaut et de F. Teisserenc dans les travaux cités dans la note suivante.

24. Voir en particulier Owen, 1973; Rowe 1995, p. 210-212, n. ad loc.; Lane 1998, p. 70-75; Pender 2000, p. 47-60 ; Dixsaut 2001, p. 267-284; Teisserenc 2010, p. 167-183. Je reprends ici pour l'essentiel l'interprétation que j'ai soutenue dans Delcomminette 2000, p. 239-242. 
- Certains êtres, «les plus grands et les plus précieux », n'ont en revanche pas ce «privilège », mais ne peuvent être manifestés que par le logos. Ces êtres incluent, au moins à titre de partie, les incorporels, qui correspondent très clairement aux Idées en général, et non pas seulement aux plus importantes d'entre elles. De fait, comme nous l'a appris Socrate dès le Phédon (99d-100a), la seule manière d'étudier les Idées est de se tourner vers les logoi plutôt que vers le sensible, et ce faisant, ce ne sont pas leurs simples images, mais leur vérité même que l'on pourra atteindre. C'est pourquoi il convient de s'exercer à donner le logos de chaque chose, et ici se trouve la justification de la longueur de l'analyse du tissage: il s'agissait d'un entraînement, destiné à nous préparer à donner le logos en vue de l'examen de l'art politique. Bien entendu, un tel entraînement n'a de sens et de pertinence que si ce à quoi il s'applique est de même nature que l'objet de la recherche-en l'occurrence, une Idée ; et de fait, comme je l'ai déjà dit plus haut, l'art du tissage n'est pas plus «sensible » que l'art politique ${ }^{25}$. Le paradigme et ce dont il est le paradigme doivent donc appartenir au même plan ontologique afin de réclamer un traitement méthodologique analogue.

- Reste que «pour tout sujet», qu'il soit sensible ou intelligible, la méthode paradigmatique réclame que l'on s'appuie sur des «petites choses » avant de passer aux grandes - bref, que l'on commence par les cas plus faciles et moins importants avant de s'attaquer aux plus difficiles et plus fondamentaux (voir déjà le texte de Sophiste, 218c-e cité plus haut). En aucun cas, cela ne peut signifier qu'il faille commencer par s'entraîner sur les choses qui présentent des ressemblances sensibles avant de passer à celles qui n'en ont pas, car la méthode à appliquer dans le premier cas ne nous serait d'aucune utilité pour le deuxième. Mais au sein du plan où l'on se meut, qu'il soit sensible ou intelligible, il faut partir de ce qui apparaît comme plus facile à celui à qui s'adresse le paradigme pour s'élever jusqu'au plus difficile - d'une syllabe que le jeune enfant connaît à une syllabe qu'il ne connaît pas, ou du tissage au politique.

- Reste que dans tous les cas, cette pratique a pour visée finale, non pas la résolution du problème ponctuel qui fait l'objet de l'examen immédiat, mais l'entraînement, la pratique, afin de devenir soit «meilleur

25. V. Goldschmidt voit d'ailleurs bien la difficulté de sa propre position, puisqu'il écrit : « Les techniques (...) ne sont pas, en toute rigueur, sensibles. Mais elles aussi ont des "ressemblances naturelles, sensibles et faciles à percevoir". » (Goldschmidt 1947, p. 59.) Resterait toutefois à montrer (1) comment cette position peut être réconciliée avec l'ensemble de son interprétation et (2) comment quelque chose de non sensible peut avoir des « ressemblances naturelles, sensibles et faciles à voir ». De toute façon, on ne voit pas pourquoi l'art politique serait moins dans ce cas que l'art du tissage. 
grammairien » soit «meilleur dialecticien ». En ce sens, on peut dire que c'est l'ensemble de la recherche concernant le politique qui constitue le paradigme de toute recherche dialectique, non pas en ce qu'elle en fournirait le plan immuable, mais au contraire en ce qu'elle montre par l'exemple la capacité d'inventivité et d'adaptation sans faille dont doit faire preuve le dialecticien aux prises avec un problème quel qu'il soit. Telle est la justification ultime de la longueur de l'analyse du tissage : plus celle-ci était élaborée - et donc, plus le modèle utilisé était construit -, plus elle était susceptible de contribuer au développement de nos capacités dialectiques. Telle est également la justification finale du Politique lui-même, avec tous ses tours et détours qui en ont dérouté plus d'un, à commencer par l'Étranger et le jeune Socrate eux-mêmes.

(4) Enfin, quatrièmement, tout comme les analogies du Gorgias et de la République, les paradigmes du Sophiste et du Politique donnent une orientation générale à la recherche qui suit. Cette fonction du paradigme est, selon moi, clairement subordonnée aux trois autres, et en particulier à la première qui est la plus fondamentale; pourtant, c'est celle sur laquelle de nombreux commentateurs insistent essentiellement, voire exclusivement. Déjà V. Goldschmidt consacrait trente-deux pages de son ouvrage à la fonction du paradigme qu'il intitulait « découverte des ressemblances », contre sept seulement à sa fonction d'exercice. Cet accent me semble inverser celui du texte de Platon. Certes, on ne peut nier que la division visant à définir la pêche à la ligne fournit un guide pour les divisions ultérieures concernant le sophiste, qui s'y rattacheront toutes d'une manière ou d'une autre; mais elle n'a pas été tout d'abord introduite à cette fin : c'est là un bénéfice accessoire d'un choix présenté comme plus ou moins hasardeux par l'Étranger ( $c f .218 \mathrm{e})$, et dont la ressemblance avec l'objet principal de la recherche ne lui apparaît qu'après coup (221d; voir toutefois $219 \mathrm{a})$. On peut même douter qu'il s'agisse réellement d'un bénéfice, du moins immédiat, puisque c'est ce rapprochement qui va conduire les interlocuteurs sur de fausses pistes, ce qui entraînera le long détour par la question de l'image, de l'erreur et de la communauté des genres afin de permettre une définition enfin satisfaisante. De même, dans le Politique, la désinvolture avec laquelle l'Étranger propose de choisir le paradigme du tissage « si nous n'avons rien d'autre sous la main $\gg(279 b)$ n'est pas feinte: de fait, n'importe quel autre art aurait pu faire l'affaire, pour autant qu'il soit lui aussi confronté à des « rivaux » au sein de sa sphère d'activité, dans la mesure où ce qui nous sert de paradigme est avant tout la méthode qu'on lui applique. Bien entendu, le politique est lui-même décrit à la fin du dialogue sur le modèle du tisserand, et à ce titre au moins, il est difficile d'imaginer choix 
plus judicieu ${ }^{26}$; mais il s'agit là d'une application supplémentaire du paradigme, pris comme «modèle » de l'activité même du politique, qu'il ne faut pas confondre avec l'application proprement dialectique qui précède les deux applications étant d'ailleurs clairement distinguées dans le texte (cf. $287 \mathrm{a}-\mathrm{b}$ et $305 \mathrm{e})^{27}$. Certes, on peut douter que l'Étranger n'ait pas prévu cette utilité supplémentaire des paradigmes qu'il propose, et on ne peut douter que Platon, lui, l'ait prévue ; mais cela est simplement dû à la construction littéraire extrêmement complexe de ces œuvres, et ne doit pas nous faire perdre de vue l'indépendance de ces deux aspects du paradigme, ni, surtout, ce qui en constitue la fonction essentielle, à savoir mettre au jour une méthode dialectique susceptible d'être appliquée à différents sujets.

Tel est à mes yeux le cœur de la pensée paradigmatique de Platon. Comme j'ai essayé de le montrer, on en trouve l'annonce dès les premiers Dialogues, dans l'usage dialectique des exemples, puis dans l'usage des analogies. Sans pour autant se confondre, ces différents procédés reposent tous sur une conception fondamentale : la dialectique, en tant que science suprême qui étudie les Idées, ne peut être décrite de l'extérieur, mais peut seulement se pratiquer. Cette pratique se prépare, non pas en commençant par se tourner vers le sensible, ce qui ne pourrait lui être d'aucune utilité - ou, pire, ne ferait que la ralentir ou l'empêcher -, mais en se tournant vers des cas tout aussi intelligibles - en tant que rapports ou structures, c'est-à-dire ensembles de rapports -, quoique « plus faciles », le but ultime étant de rendre le dialecticien capable d'examiner n'importe quel sujet. On voit donc que le paradigmatisme de Platon, loin d'impliquer un quelconque retour du sensible au sein même de l'activité dialectique, est intrinsèquement lié à sa conception de la dialectique comme activité de la pensée pure, et à l'identification qu'il opère entre la science et la méthode - la dialectique n'étant subordonnée à aucun objet particulier de recherche, mais utilisant tout objet qui se présente comme prétexte à son propre déploiement.

26. Pour une analyse approfondie des raisons qui ont pu pousser l'Étranger à choisir l'art du tissage comme paradigme, voir El Murr 2002.

27. Sur ces deux applications du paradigme du tissage, voir Delcomminette 2000, p. 273-320. 


\section{BIBLIOGRAPHIE}

BURnYEAT, M.F. 1977 : «Examples in epistemology: Socrates, Theaetetus and G.E. Moore », Philosophy, 52 (1977), p. 381-398.

Canto-Sperber, M. 1993a : Platon, Ménon, Traduction inédite, introduction et notes, Paris, 1993 [ $\left.{ }^{1} 1991\right]$ (GF, 491).

- 1993b : Platon, Gorgias, Traduction inédite, introduction et notes, Paris, 1993 [ $\left.{ }^{1} 1987\right]$ (GF, 465).

Delcomminette, S. 2000 : L'inventivité dialectique dans le Politique de Platon, Bruxelles, 2000 (Cahiers de philosophie ancienne, 16).

- 2006: Le Philèbe de Platon. Introduction à l'agathologie platonicienne, Leyde, 2006 (Philosophia antiqua, 100).

- 2008: «Faculté et parties de l'âme chez Platon », Plato. The Internet Journal of the International Plato Society, 8 (2008), p. 8-13, URL: http://gramata.univparis1.fr/Plato/article83.html.

DIÈS, A. 1925 : Platon, Le Sophiste, Texte établi et traduit par -, Paris, 1925 (Collection des Universités de France).

DiXsAut, M. 2001 : Métamorphoses de la dialectique dans les dialogues de Platon, Paris, 2001 (Bibliothèque d'histoire de la philosophie).

DORION, L.-A. 1997 : Platon, Lachès; Euthyphron, Traduction inédite, introduction et notes, Paris, 1997 (GF, 652).

- 2004 : Platon, Charmide; Lysis, Traduction inédite, introduction et notes, Paris, 2004 (GF 1006).

El MURR, D. 2002: «La symplokè politikè : le paradigme du tissage dans le Politique de Platon, ou les raisons d'un paradigme 'arbitraire' », Kairos, 19 (2002), p. 49-95.

Ferrari, G. R. F. 2003 : City and Soul in Plato's Republic, Sankt Augustin, 2003 (Lecturae Platonis, 2).

GOLDSCHMIDT, V. 1947 : Le Paradigme dans la dialectique platonicienne, Paris, 1947.

LANe, M. 1998 : Method and Politics in Plato's Statesman, Cambridge, 1998 (Cambridge Classical Studies).

LLOYD, G. E. R. 1966 : Polarity and Analogy. Two Types of Argumentation in Early Greek Thought, Cambridge, 1966. [Réimpr. Indianapolis, 1992.]

Mattéi, J.-F. 1996 : Platon et le miroir du mythe. De l'áge d'or à l'Atlantide, Paris, 1996 (Thémis. Philosophie).

OWEN, G. E. L. 1973 : « Plato on the undepictable », dans E. N. Lee, A. P. D. Mourelatos et R. M. Rorty (éd.), Exegesis and Argument. Studies in Greek Philosophy presented to G. Vlastos, Assen, 1973 (Phronesis: a journal for ancient philosophy. Supplementary volume, 1), p. 349-361.

PAChet, P. 1993 : Platon, La République: Du régime politique, Traduction de —, Paris, 1993 (Folio/Essais, 228).

Pender, E. E. 2000 : Images of Persons Unseen. Plato's Metaphors for the Gods and the Soul, Sankt Augustin, 2000 (International Plato studies, 11).

RABBÅS, Ø. 2004: «Definitions and paradigms : Laches's first definition », Phronesis, 49 (2004), p. 143-168.

Robin, L. 1929 : Platon, Le Banquet. Texte établi et traduit par —, Paris, 1929 (Collection des Universités de France).

Robinson, R. 1953 : Plato's Earlier Dialectic, Second Edition, Oxford, 1953.

Rowe, C. J. 1995 : Plato, Statesman, ed. with an introd, transl. and commentary, Warminster, 1995 (Classical Texts).

TeIsSERENC, F. 2010 : Langage et image dans l'auvre de Platon, Paris, 2010 (Tradition de la pensée classique). 